\title{
LES VIES DE PHILOSOPHES DE DIOGÈNE LAËRCE: UNE RÉFLEXION SUR L'HISTOIRE DE LA PHILOSOPHIE
}

\author{
Christelle Veillard \\ Université de Paris $\mathrm{X}$
}

\begin{abstract}
Diogenes Laertius' Lives and opinions of Eminent Philosophers is one of the monumental works of ancient philosophy, an inestimable source of biographical and doctrinal information. For a certain time, little value was assigned to the work, since Diogenes Laertius used to be considered someone who was not able to comprehend doctrines, had the unpleasant habit of compiling them out of order and was fond of uninteresting anecdotes. Diogenes is commonly presented as a great scholar, passionate about philosophy, who copied without understanding what was copied - someone not to be entirely trusted. However, contemporary studies have shown that such point of view must be corrected through the analysis of the structure of Lives, in spite of the still present disagreement over the choices the author made when reorganizing the works he compiled, both in terms of the scope and meaning of such choices. Two interpretative threads are identified: one being deflationary, the other, inflationary. This article tests the validity of the inflationary interpretation, and the investigation will concern two main questions: 1) Does the text show traces of the author's personality?; 2) Is the text the result of a personal conception of the history of philosophy?
\end{abstract}

Keywords: Diogenes Laertius, deflationary interpretation, inflationary interpretation.

Resumo: As vidas e doutrinas dos filósofos ilustres de Diógenes Laércio é uma das obras monumentais da filosofia antiga, fonte inestimável de informações biográficas e doutrinais. Durante um certo período, pouco valor foi atribuído à obra, pois Diógenes Laércio era considerado alguém sem maior capacidade para compreender as doutrinas, que possuía 0 hábito desagradável de compilá-las sem ordem, as doutrinas, e afeito a anedotas desinteressantes. Diógenes é comumente apresentado como um grande erudito, apaixonado pela filosofia, mas que copia sem compreender o que copia, ao qual se deve atribuir uma confiança limitada. Estudos contemporâneos mostram, entretanto, que este ponto de vista deve ser corrigido, a partir da análise da estrutura do texto das Vidas, embora as escolhas que fazia ao reorganizar as obras as quais compilava ainda cause desacordo, seja no que diz respeito ao alcance, seja no que diz respeito à significação destas escolhas. Duas linhas 
interpretativas podem ser identificadas: uma deflacionista, outra inflacionista. A intenção deste artigo é testar a validade da interpretação inflacionista, sendo que a investigação concernirá a duas questões principais: 1) 0 texto mostra os traços da personalidade do autor?; 2) o texto é fruto de uma concepção particular acerca da história da filosofia ?

Palavras-chave: Diógenes Laércio, interpretação deflacionista, interpretação inflacionista.

\section{Introduction}

Les Vies et doctrines des philosophes illustres rédigées par Diogène Laërce sont incontestablement l'un des monuments de la philosophie antique. Elles sont pour nous une source inestimable de renseignements biographiques et doctrinaux sur les philosophes anciens, dont certains noms ou certaines œuvres ne sont connues que par leur intermédiaire. L'exemple le plus fameux est évidemment celui des Lettres d'Épicure, qui nous sont providentiellement transmises, in extenso, par le livre X. Les Vies proposent par surcroît un tableau ordonné de la philosophie, des Sept Sages jusqu'au I ${ }^{\text {er }}$ s. après J.-C $\mathrm{C}^{1}$. Ce monument a fait l'objet d'interprétations contrastées. Il fut un temps où l'on faisait peu de cas de Diogène Laërce, soulignant ses piètres capacités à comprendre certains points de doctrine, sa fâcheuse habitude de compiler sans ordre des extraits en dehors de leur contexte d'origine, son goût pour les anecdotes sans intérêt, anecdotes qui, par ailleurs, se démentent parfois les unes les autres ${ }^{2}$. La manière habituelle de présenter Diogène est de le peindre comme un très grand érudit féru de philosophie, qui ne comprend pas toujours ce qu'il copie et à qui l'on doit par conséquent accorder une confiance très limitée. L'exemple de la notice consacrée à Aristote, aussi brève qu'inexacte, jetterait ainsi le discrédit sur la totalité des Vies. L'ouvrage est-il pour autant sans ordre ni sens ? Des études récentes ${ }^{3}$ ont montré, à partir de l'analyse de la structure même du texte, que ce jugement doit être corrigé. Il est devenu clair aujourd'hui que si Diogène Laërce s'inscrit dans une longue

\footnotetext{
1 L'ouvrage tel qu'il nous est parvenu ne va pas au-delà du iie s. av. J.-C., mais la table des matières que nous avons conservée indique qu'il devait se poursuivre jusqu'au ier s. apr. J.-C. (cf. la note 7).

2 Citons l'exemple de Xénophon, dont il est dit qu'il fut dans la force de l'âge vers 401-400 en DL, II. 55, tandis que plus loin, en DL, II. 59, Diogène dit qu'il fut dans la force de l'âge vers 424-420.

${ }^{3}$ Cf. entre autres: Diogene Laerzio storico del pensiero antico, Elenchos, 7, 1-2, Napoli, Bibliopolis, 198; HAASE, W; TEMPORINI, H. (eds). Aufstieg und Niedergang der Römischen Welt, II, 36, 6. Berlin - New York: W. de Gruyter, 1992; MEJER, J. Diogenes Laertius and his hellenistic background. Hermès, 40, Wiesbaden, Steiner, 1978; GoulET-CAzÉ, M.-O. (dir.), Diogène Laërce. Vies et doctrines des philosophes illustres, ["Classiques Modernes"]. Paris: Le Livre de Poche, 1999; DORANDI, T. Le stylet et la tablette: dans le secret des auteurs antiques. Paris: Les Belles Lettres ["L'âne d'or, n 12"], 2000.
} 
tradition de doxographes et qu'il compile comme tous les autres les ouvrages de ses nombreux prédécesseurs, il ne le fait pas tout à fait au hasard mais opère des choix, des réorganisations visibles, sinon explicites.

Les commentateurs restent toutefois en désaccord quant à la portée et à la signification de ces choix. Nous pouvons identifier deux lignes interprétatives, auxquelles nous avons, pour plus de commodité, appliqué deux étiquettes.

La première ligne interprétative peut être qualifiée de déflationniste. Elle suppose que Diogène Laërce est un érudit qui se contente de compiler des matériaux disparates, ajoutés les uns aux autres au fur et à mesure de leur découverte. Deux conséquences majeures s'ensuivent: 1). son œuvre est sans ordre ni dessein particulier; 2). elle ne porte pas la marque de l'individualité de son auteur: même lorsque l'on trouve des commentaires qui semblent plus personnels sur les doctrines ou sur les philosophes, et qui pourraient être le signe d'une intervention personnelle du laërtien, on peut les expliquer en supposant que Diogène, même là, se contente de recopier des commentaires qu'il a trouvés dans les sources qu'il utilise. L'œuvre de Diogène Laërce possède indiscutablement ce caractère disparate et déconcertant, qui relève de la nature même des ouvrages de type doxographique: ce sont avant tout des compilations dans lesquelles il est malaisé de déterminer les apports respectifs du compilateur et de ses sources. Il est tentant de conclure à une intervention minimale du compilateur, qui n'a fait que "copier-coller" des morceaux épars, se contentant de composer les phrases de transition qui les articulent. D'où cette conclusion: l'ouvrage n'aurait ni auteur ni ancrage historique, la structure qui nous est présentée ne relèverait d'aucune conception précise de l'histoire de la philosophie ${ }^{4}$.

\footnotetext{
${ }^{4}$ C'est notamment la position que semble adopter R. GoULET, "Les Vies de philosophes dans l'Antiquité tardive", dans ID., Etudes sur les Vies de philosophes de l'Antiquité tardive. Paris: Vrin ["Textes et Traditions"], 2001, p. 3-63. II souligne que les informations contenues dans les doxographies sont soumises à plusieurs "axes de déformation", dus tant au projet individuel du doxographe qu'au matériel plus ou moins fiable qu'il utilise, identifiant en particulier un axe dit "idéologique" qui correspond "à la finalité qui est imposée à la biographie" (p. 14). Analysant le souci d'édification morale des biographies, il remarque que "bien peu de nos Vies de philosophes échappent à cette perspective, sauf peut-être les Vies et doctrines des philosophes illustres de Diogène Laërce. [...] Les dix livres qui les composent sont inspirés par la curiosité encyclopédique plus que par le souci d'édification morale et ne sont qu'une compilation d'ouvrages plus anciens qui présentaient sans doute déjà les mêmes caractères. Dans le texte de Diogène viennent se déposer les alluvions, anciennes ou récentes, d'innombrables courants biographiques et doxographiques. Dans un tel capharnaüm, où sont juxtaposées des traditions souvent contradictoires, on chercherait en vain un point de vue cohérent sur les philosophes et l'histoire de la philosophie" (p. 15). II est vrai que l'ouvrage laërtien est quelque peu désordonné. Est-ce à dire
} 
La seconde ligne interprétative pourrait à l'inverse être dite inflationniste: elle consiste à faire apparaître la personnalité de l'auteur, mais surtout à soutenir que les Vies ont été composées en vue d'un objectif bien précis, qui déborde de loin le cadre de la simple collection. Cette interprétation est le moyen de redonner une cohérence à un ensemble qui paraît disparate, mais également d'attribuer à Diogène la responsabilité de la composition de l'ouvrage: ainsi n'est-ce pas seulement parce qu'il a eu accès à telle ou telle source, qu'il en use dans son ouvrage; s'il l'utilise, c'est qu'il a une bonne raison de le faire. De même, la structure de l'ouvrage, les redondances et les commentaires, loin d'être le résultat accidentel de compilations hasardeuses, pourraient participer d'un projet d'écriture particulier. Nous pouvons par exemple remarquer qu'il existe un décalage entre le contenu de l'exposé laërtien et le moment supposé de son écriture, à savoir la première moitié du $\mathrm{III}^{\mathrm{e}} \mathrm{s}$. apr. J.-C ${ }^{5}$. Diogène ne cite ni Porphyre, ni Plotin, ni aucun des représentants de la littérature chrétienne grecque ou latine (Tertullien, Hippolyte, Clément d'Alexandrie). Il ne semble pas connaitre les nouveaux aménagements du Péripatos d'Alexandre d'Aphrodise ou de l'Académie de Philon de Larisse et d'Antiochus d'Ascalon; il arrête ses explications relativement tôt, au $\mathrm{II}^{\mathrm{e}} \mathrm{s}$. av. J.-C. pour ce qui est de l'ouvrage tel qu'il nous est parvenu ${ }^{6}$. Il avait cependant traité de philosophes appartenant au I ${ }^{\mathrm{er}}$ s. apr. J.-C., d'après la table des matières reflétant le contenu des Vies que nous avons conservée, par laquelle nous savons précisément qu'une

cependant que cette compilation n'est réalisée selon aucun plan ni intention personnels ? Parce que Diogène recopie ses devanciers et qu'il semble reprendre sans distinction tout ce qu'il trouve, il est soupçonné n'avoir aucun point de vue, les adoptant tous. De même, lorsqu'il commente les défauts ou vertus de certains philosophes, ses commentaires sont vidés de toute intentionnalité, puisque l'on suppose qu'ils émanent de ses sources. Qu'il admire, condamne, raille ou célèbre, "en tout cela Diogène se laisse guider par ses sources, empruntant éloges et railleries à ses devanciers" (p. 16). Cela revient presque à dire que les Vies n'ont pas d'auteur, ou un auteur si transparent qu'il en devient négligeable eu égard à la compréhension de son texte.

${ }_{5}$ La question de l'ancrage historique de l'œuvre est difficile: il ne paraît pas possible de situer exactement le moment où les Vies ont été écrites, ni le lieu où elles le furent. De la même façon, nous ne savons presque rien de Diogène Laërce. Si cet obstacle est de taille, il n'est pas rédhibitoire. La lecture des Vies nous apprend que l'auteur connaissait Sextus Empiricus (IX. 87; 116). Selon Mgr P. Descourtieux, il écrit peu de temps après Clément d'Alexandrie, qui compose lui sous Sévère, c'est-àdire entre 193 et 211, selon les indications d'Eusèbe de Césarée, Histoire Ecclésiastique VI. 6 (Cf. Clément d'Alexandrie. Les Stromates, VI, texte et traduction Mgr P. Descourtieux, Cerf ["Sources Chrétiennes" - n 446], 1999; introduction p. 19).

${ }^{6}$ Pour le Péripatos, le livre se termine par Démétrios de Phalère (mort vers 283 av. J.-C.) et Héraclide (dont la présence dans ce livre est douteuse); pour l'Académie, par Clitomaque, scolarque en 127 av. J.-C. 
partie du livre VII consacré aux stoïciens a été perdu. Cet Index Locupletior ${ }^{7}$ indique qu'après Chrysippe il était question de nombreux stoïciens (parmi lesquels Panétius, Posidonius, Hécaton); la liste se termine par la mention de Cornutus, dont nous savons qu'il écrivit au ${ }^{\mathrm{er}} \mathrm{s}$. apr. J.-C. Les déflationnistes pourront supposer que ces lacunes chronologiques s'expliquent par un manque de documentation: éloigné des grandes bibliothèques au moment de composer son ouvrage, Diogène aurait manqué de matériel plus récent ${ }^{8}$. Les inflationnistes supposeront, au contraire, que ces lacunes sont le résultat d'un choix, choix potentiellement visible dans la manière dont Diogène Laërce a agencé son texte?

${ }^{7}$ Cette table des matières, que l'on trouve dans le codex Parisinus graecus $1759(P)$, de la fin du XIIIe S., a été éditée et étudiée par T. DORANDI, "Considerazioni sull'Index Locupletior di Diogene Laerzio". Prometheus 18, 1992, p. 121-126. L'édition de l'Index se trouve p. 122.

${ }^{8}$ Deux hypothèses ont été proposées: Diogène est dit "Diogène Laërce" car il se trouve à Laërtès, petite ville de Cilicie (cf. M.-O. GoulET-CazÉ, "Introduction générale" dans ID. (dir.), Diogène Laërce. Vies et doctrines des philosophes illustres, La Pochotèque, Le Livre de Poche, ["Classiques Modernes"], 1999, p. 9-27, en particulier p. 11-12. La seconde hypothèse est qu'il se trouve à Nicée, d'après une indication de DL, IX. 109: Apollonidès de Nicée est "l'un des nôtres" d'après la lecture de J. J. REISKE dans H. DIELS, "Reiskii animadversiones in Laertium Diogenem". Hermes, 24, 1889, p. 302-325, ici p. 324; de même J. MANSFELD, "Diogenes Laertius on Stoic Philosophy". Elenchos, 7, 1986, p. 297-382, ici p. 300301. Qu'il s'agisse donc de Laërtès ou de Nicée, il écrit dans une région reculée de l'Empire et ne dispose peut-être pas de documentation récente. D'où un repli possible sur les manuels classiques et sur des sources comprises majoritairement entre le troisième et le premier siècle av. J.-C., par exemple, pour le livre VII: Apollodore de Séleucie, par l'intermédiaire de Dioclès de Magnésie, Antigone de Caryste, Hippobote, etc.

9 David Hahm a étudié dans cette optique la doxographique éthique attribuée à Arius Didyme et transmise par le chapitre VII du livre II des Éclogues de Stobée. Cf. D. E. HAHM, "The Ethical Doxography of Arius Didymus". ANRW II. 36. 4, HAASE (éd.), Walter de Gruyter, Berlin/NewYork 1990, p. 2935-3055; p. 2993 sq. Ses conclusions sont les suivantes: chaque doxographie est ordonnée en fonction d'une perspective propre, qui réorganise les éléments traditionnels de l'école. Par exemple, le passage stoïcien est structuré selon la division des êtres (biens, maux, indifférents) puis des actions (morales, immorales, indifférentes). Le passage aristotélicien est fondé sur la nature de la vertu, son développement et son expression dans la vie morale. Les trois doxographies sont par ailleurs intégrées dans un schéma plus grand, chaque perspective de l'une complétant celles des deux autres. Ainsi l'éthique tout entière est-elle exposée sous des perspectives complémentaires, par une sorte de procédé polyphonique selon les termes de Hahm: "chaque exposé, en présentant les opinions particulières de chaque école à partir d'une perspective particulière, intègre la perspective de l'autre comme un thème subordonné, et ainsi crée un lien invitant le lecteur" à voir les doxographies comme complémentaires. L'objectif de l'auteur est de nous faire comprendre que tous les philosophes disent la même chose, mais sous des points de vue différents, ce qui fait lire la philosophie non comme un ensemble de positions discordantes, mais au contraire comme l'expression d'une "polyphonie harmonique" (p. 3000 notre traduction). Notons que l'hypothèse est séduisante, même si elle ne fonctionne pas pour tous les passages du texte. Sur cette question, cf. également Cristina Viano, "L'Epitomê de l'éthique stoïcienne d'Arius Dydime (Stobée, Eclog. II. 7. 57. 13 - 116. 18)" dans G. Romeyer Dherbey (dir.) \& J.-B. Gourinat (éd.). Les Stoïciens, Paris, Vrin, 2005, p. 335-355. 
Nous pourrions trancher entre les deux interprétations proposées si nous savions de quelles sources disposait Diogène Laërce ${ }^{10}$. Ne possédant pas sa bibliothèque, il nous reste à scruter le texte, que nous nous proposons de relire en partant des éléments qui pourraient rendre sensible la présence du laërtien dans son œuvre. Notre principe méthodologique est de prendre au sérieux ce que dit le texte, sa structure comme les choix qu'il manifeste. Ce que nous cherchons par conséquent à tester, c'est plus précisément la validité de l'interprétation dite inflationniste. Notre enquête portera sur deux questions principales: 1). Le texte porte-t-il la trace de la personnalité de son auteur ? 2). Le texte est-il empreint d'une conception particulière de l'histoire de la philosophie?

\section{Les Vies ont-elle un auteur?}

Le premier élément notable est la présence d'épigrammes ${ }^{11}$ composées par Diogène Laërce et dont il a parsemé son récit. Elles traitent principalement de la mort des personnages évoqués.

Une question préalable doit être posée: quel est le statut et la fonction des épigrammes au sein des Vies? De manière déflationniste, on pourrait dire que l'insertion de ces poèmes avait une seule fonction, celle d'amuser, de divertir, en disant sous une forme plus percutante ce qui avait déjà été dit auparavant. Pourtant, lorsque Diogène cite plusieurs versions de la mort d'un philosophe, seule l'une d'entre elles est l'objet d'épigramme: il s'agit de l'une des versions rationnelles qu'il a fournies, éclipsant ainsi les récits surnaturels ${ }^{12}$. Les épigrammes ont par ailleurs une unité de signification: Diogène admire Platon et Socrate, méprise le suicide considéré comme une lâcheté, dénonce l'ivrognerie, etc. Ces régularités indiquent qu'elles expriment des convictions laërtiennes, et ne sont pas l'imitation servile d'autres poètes ou des sources utilisées.

\footnotetext{
10 Non pas quelles sources il a utilisées, ce dont nous pouvons avoir une idée, même vague, mais de quels ouvrages il avait connaissance. Il est clair que, sauf à retrouver sa bibliothèque, il est impossible de le savoir.

11 On trouve en DL, I. 63 l'annonce du contenu du Pammétros: "Nous avons nous aussi écrit une épigramme dans notre Pammétros déjà citée où j'ai traité de la fin de tous les hommes illustres dans tous les mètres et tous les rythmes, sous forme d'épigrammes et de chants". M. GIGANTE, "Biografia e dossografia in Diogene Laerzio", Elenchos, 7, 1986, p. 7-102, suppose p. 38 que le livre I traite de la mort tandis que le livre II, écrit en même temps que les Vies, traite des doctrines.

12 Voir les exemples donnés $p$. 11, en particulier la note 39.
} 
Le contenu des épigrammes nous révèle enfin un point essentiel: la mort est le moment crucial où sont mises à jour les tendances les plus profondes de l'individu dont on a rédigé la vie. Diogène Laërce semble ici adopter un principe proche du proverbe fameux selon lequel il ne faut jamais dire de quelqu'un qu'il est heureux avant qu'il ne soit mort. Ici, il semble plus précisément signaler que nous ne pouvons avoir une idée adéquate de la nature profonde de chacun qu'après sa mort, une fois affronté cet événement qui teste la résistance tant des principes théoriques soutenus de son vivant, que la force intellectuelle et émotionnelle des individus qui ont tous prôné et adopté des comportements vertueux et droits. Nous remarquons en effet deux types de critiques. D'une part, une critique comportementale: l'individu mourant est simplement terrifié et, n'ayant pas une attitude très digne, se trouve confronté au ridicule. C'est le cas de Carnéade ${ }^{13}$. Ou bien, c'est son comportement inadapté qui a provoqué sa mort, par exemple l'ivrognerie pour Arcésilas et Lacydès ${ }^{14}$. Leur mort prouve qu'ils n'étaient que des simulacres de sages. On trouve un second type de critique plus radicale, qui souligne la possible distorsion entre les principes philosophiques soutenus et leur mise en application, surtout au moment de la mort, qui fonctionne comme un révélateur. Par exemple, Bion est raillé pour avoir renié au moment suprême ses positions antérieures sur les dieux ${ }^{15}$; Ménippe, pour s'être pendu parce qu'il avait perdu tous ses biens, ce qui est le comble pour un cynique ${ }^{16}$.

$\mathrm{Si}$ les épigrammes sont les traces évidentes de l'intervention personnelle de Diogène, elles n'en constituent pas pour autant le seul lieu où Diogène Laërce s'exprime. Ses interventions sont également visibles dans la construction de l'ouvrage: il annonce dans le Prologue ${ }^{17}$ un plan qu'il suit plus ou moins. Le texte porte les traces de remaniements ultérieurs ${ }^{18}$ ainsi que de transitions et de justifications de l'ordre présenté ${ }^{19}$. Enfin, nous

\footnotetext{
${ }^{13}$ DL, IV. 64-66: "Seul un ignorant ne sait pas comment il a craint de mourir".

14 Arcéslias est dit avoir "outragé les Muses" (DL, IV. 44); Lacydès est attrapé par la pointe des pieds par un Bacchus inopportun qui l'a ainsi entraîné dans l'Hadès (DL, IV. 61).

${ }_{15} \mathrm{DL}, \mathrm{IV} .54$.

${ }^{16}$ DL, VI. 99-100.

${ }_{17} \mathrm{DL}, \mathrm{I} .13-15$.

${ }_{18} \mathrm{DL}$, VI. 81; VI. 19 à comparer avec II. 47. Sur toutes ces questions, voir M.-O. GoULET-CAZÉ et M. NARCY, "Introduction au livre II", dans M.-O. GoulET-CAZÉ (dir.), Diogène Laërce. Vies et doctrines des philosophes illustres, La Pochotèque, Le Livre de Poche, ["Classiques Modernes"], 1999, p. 161-205, en particulier les pages 164-165 et 202.

${ }_{19}$ DL, I. 20; VI. 19 et 105; VII. 38, 160 et 167.
} 
trouvons çà et là de petits commentaires indéniablement personnels ${ }^{20}$ La construction d'ensemble est apparemment due à deux éléments principaux: Diogène suit d'une part la division présentée par Sotion dans ses Successions, il adopte d'autre part l'hypothèse propre à la tradition d'Apollodore de Séleucie (transmise par Dioclès de Magnésie) qui voit une filiation entre cynisme et stoïcisme, et fait d'Antisthène le fondateur de l'école cynique. Cela est rendu possible par l'adoption de la thèse selon laquelle le cynisme est bien une école, contrairement à ce que soutient Hippobote ${ }^{21}$. Nous reviendrons sur ce point primordial un peu plus loin.

Par ailleurs, si Diogène Laërce dispose de nombreuses sources divergentes, il ne les mentionne pas de la même façon: les sources qui ne reçoivent pas son suffrage, par exemple Hippobote ${ }^{22}$, occupent dans le texte une position marginale. Pourquoi les citer alors? Si toutes étaient citées de la même façon, nous pourrions en conclure au rassemblement de tous les renseignements disponibles. Cependant on note ici un choix, une hiérarchie de présentation, qui indique que si toutes les sources possèdent par définition un égal droit de cité, toutes cependant n'ont pas la même valeur aux yeux de l'auteur ${ }^{23}$. Pour résumer, Diogène Laërce choisit de favoriser les liens de filiation entre les écoles, il sélectionne et hiérarchise ${ }^{24}$ ses sources en fonction des traditions qu'elles représentent. On note également qu'il passe volontairement sous silence des éléments contemporains pourtant connus ${ }^{25}$.

Si l'exposition des différentes traditions philosophiques se fait toujours de manière neutre et non critique, cela ne veut pas dire pour autant

\footnotetext{
20 II dit au sujet des ouvrages d'Aristote: "J'ai trouvé judicieux d'en donner la liste, à cause de l'excellence de cet homme dans tous les genres de discours" (DL, V. 21); à propos des ouvrages de Théophraste: "Eux aussi, j'ai jugé légitime d'en donner la liste parce qu'ils regorgent d'excellence en tous domaines" (DL, V. 42).

${ }^{21} \mathrm{DL}, \mathrm{I} .19$.

22 Voir par exemple le début du livre VII: VII. 2; VII. 31 et 24.

${ }^{23}$ On peut penser de la même façon que, quand bien même Diogène recopierait dans sa source les commentaires laudatifs sur les lois de Solon par exemple (DL, I. 55-56), ou sur Stilpon (DL, II. 112), il n'en reste pas moins que ces derniers sont le signe d'un accord de l'auteur. II serait pour le moins improbable qu'il recopiât des commentaires avec lesquels il se trouverait en désaccord.

24 Voir par exemple les deux traditions reliant ou non le cynisme au stoïcisme: sur ce point, Dioclès de Magnésie, Apollodore de Séleucie, Néanthe de Cyzique (DL, I. 20; VI. 13; VII. 121) s'opposent à Hippobote (DL, I. 19; VI. 85; VII. 25), Apollodore le Chronographe et Philodème de Gadara (cf. Philodème de Gadara, PHerc. 339, col. XIII p. 101 éd. Dorandi, dans T. DoRANDI, "Filodemo. Gli Stoici (PHerc. 155 e 339)", Cronache Ercolanesi, 12, 1982, p. 91-133).

${ }_{25}$ Nous renvoyons aux pages 47, 48 et 50 de l'article de M. GIGANTE, "Biografia e dossografia in Diogene Laerzio", Elenchos, 7, 1986, p. 7-102.
} 
que toute tentative de l'esprit pourra trouver sa place dans l'exposé laërtien, qui semble procéder de choix précis.

\section{Les Vies: une histoire de la philosophie anti-dogmatique}

Le texte contient en effet des éléments qui font sens vers une conception particulière de la philosophie et de son histoire. Le premier indice est la présence dans le texte de deux définitions concurrentes de ce qu'est une école philosophique:

Hippobote, dans son traité Sur les écoles de pensée, dit qu'il y a neuf écoles de pensée ou mouvements: l'école mégarique, l'érétriaque, la cyrénaïque, l'épicurienne, l'annicérienne, la théodoréenne, la zénonienne également appelée stoïcienne, l'académicienne antique, la péripatéticienne; mais il ne retient ni la cynique, ni l'éliaque, ni la dialectique. Car l'école pyrrhonienne, la plupart ne l'incluent pas non plus à cause de son obscurité. Certains cependant disent qu'elle est une école de pensée sous un certain rapport et qu'elle ne l'est pas sous un autre. Il semble cependant qu'elle soit une école de pensée. Nous considérons en effet comme une école de pensée celle qui suit ou semble suivre une certaine façon de penser en respectant les apparences. Selon ce principe, on appellerait à juste titre l'école sceptique une école de pensée. Si au contraire nous concevions l'école de pensée comme l'adhésion à des doctrines présentant un enchaînement, on ne saurait plus l'appeler une école de pensée, car elle ne possède pas de doctrines ${ }^{26}$.

Pour Diogène, l'école est définie par une manière commune de raisonner et non par l'adhésion à un ensemble d'opinions ou de croyances. Pour Hippobote à l'inverse, il s'agit d'un ensemble organisé de dogmes. Cette distinction est d'importance, en ce qu'elle nous signale qu'il ne faut pas comprendre l'exposé des doctrines comme des résumés de croyances à adopter mais bien plutôt comme des exemples de voies possibles, des manières différentes pour l'esprit de cheminer ${ }^{27}$. Diogène souligne d'ailleurs que son propos n'est en aucune façon de convertir à l'une d'entre elles ${ }^{28}$.

\footnotetext{
${ }^{26}$ DL, I. $19-20$ (trad. Richard Goulet). Nous retrouvons ces deux conceptions dans un passage de SeXtus EmpIRIcus, Esquisses Pyrrhoniennes, I. 16-17: l'appartenance à une école est définie soit comme l'inclination à suivre des dogmes, soit comme la décision de "suivre la voie qui suit un raisonnement déterminé", donc comme l'adoption d'une certaine manière d'utiliser sa raison.

27 La filiation supposée entre les écoles cynique et stoïcienne (DL, VI. 15, 19 et 105 doit alors être interprétée moins comme une proximité doctrinale, que comme le parcours d'une même route, l'emprunt
} 
Le second indice est le fait que les Vies de Diogène Laërce ne ressemblent guère aux ouvrages de ses contemporains. Elles ne prétendent pas convertir à un dogme philosophique particulier et s'opposent en cela aux exposés de Sextus Empiricus ( $\mathrm{II}^{\mathrm{e}}-\mathrm{III}^{\mathrm{e}} \mathrm{s}$ apr. J.-C.), qui ont pour objectif d'imposer le scepticisme comme la seule voie possible, en mettant en évidence les difficultés et contradictions des systèmes dits dogmatiques; de Clément d'Alexandrie (140-220), dont les Stromates sont une exhortation à la vraie philosophie qu'est le christianisme; de Porphyre, qui rédige son ouvrage Sur la Vie de Plotin et l'ordre de ses livres pour introduire aux Ennéades. Diogène ne fait pas de prosélytisme, mais propose une simple exposition des principales doctrines. Cela étant, tous les chemins empruntés par l'esprit humain sont-ils également dignes d'être poursuivis, et l'histoire racontée par Diogène est-elle philosophiquement neutre? Il semble bien que l'auteur nous mette en garde contre certaines voies.

La troisième caractéristique que nous croyons notable est le refus de faire de la philosophie une pratique marquée par les mystères et la révélation. Considérant l'origine de la philosophie, Diogène Laërce souligne avec force qu'elle n'est pas d'invention barbare, comme de nombreux auteurs l'ont soutenu, mais bien grecque ${ }^{29}$. Il part d'une division typiquement aristotélicienne pour écarter les Mages et autres Gymnosophistes, refusant ainsi les conceptions orientalisantes propres à son époque, qui définissent la philosophie comme une énigme, un mystère et non pas comme la reprise rationnelle des discours des $\operatorname{Sages}^{30}$. Ces derniers fondent les traditions philosophiques, mais toute interprétation "barbare" des faits, c'est-à-dire irrationnelle, mystérieuse, mystique, pythagoricienne pourrait-on dire, sera placée au second plan et désapprouvée. Cette tendance est visible si nous

d'une même voie de raisonnement: c'est pourquoi le stoïcisme constitue la voie longue d'accès à la vertu (par l'adoption de dogmes précis), tandis que le cynisme est un raccourci (cf. DL, VI. 104).

28 II mentionne la méthode de Potamon d'Alexandrie (DL, I. 21) qui consiste à prendre dans chaque école les éléments qui lui paraissent les meilleurs. Si l'on cherche à quelle école aurait bien pu appartenir Diogène, on pourrait conclure à la fois en faveur de l'éclectisme (DL, I. 21), de l'épicurisme (DL, X. 138) et du scepticisme (DL, IX. 109), ce qui montre que ce n'est pas une bonne manière de poser le problème.

29 DL, I. 3.

${ }^{30}$ Aristote soulignait la filiation entre sages et philosophes qui tous commencent par l'étonnement, la constatation de leur ignorance, mais qui diffèrent ensuite: les seconds articulent clairement et distinctement les impressions confuses qu'avaient les premiers sur la nature des choses: cf. ARISTOTE. Métaphysique, A. 4. 985 a 11 sq. 
comparons à nouveau Diogène Laërce avec ses contemporains ${ }^{31}$. L'exemple le plus marquant est la Vie d'Apollonius de Tyane rédigée par Philostrate $\mathrm{d}^{\prime}$ Athènes ${ }^{32}$, où le philosophe prend les traits de l'homme divin ${ }^{33}$ : affranchi de ses limites corporelles (il est capable d'ubiquité, de lévitation), il est la présence incarnée du dieu qui se manifeste par une aura, un scintillement de tout son être, ce qui justifie qu'on lui rende un culte de son vivant. Dans ce cadre, la philosophie est d'ordre mystérique et non plus seulement rationnel, elle ressortit plus de la révélation divine que d'un effort de la raison humaine vers la connaissance ${ }^{34}$. Cette nouvelle manière de comprendre la philosophie et le philosophe se lit dans les ouvrages de Philostrate, dans la Vie de Pythagore de Porphyre, dans la Vie pythagoricienne de Jamblique et plus tard également dans les Vies de philosophes et de sophistes d'Eunape de Sardes ${ }^{35}$.

Contre eux, Diogène Laërce aura tendance à résister contre ce que l'on pourrait appeler une vision orientalisante du philosophe; nous pouvons le constater dans sa Vie de Pythagore. Diogène ne peut pas ne pas traiter de Pythagore, en particulier parce qu'il suit la succession proposée par Sotion d'Alexandrie qui le place à la tête de la branche italique de la philosophie - la branche ionienne étant fondée par Thalès. La Vie pythagoricienne reste cependant tout à fait semblable aux autres, Diogène ne suivant visiblement

\footnotetext{
${ }^{31}$ Nous convoquerons Philostrate d'Athènes (170-245), Jamblique (250-330), Porphyre (234-301).

32 La Vie d'Apollonius de Tyane de Philostrate d'Athènes semble marquer un tournant dans l'histoire de la philosophie: cf. R. GoulET, "Les Vies de philosophes dans l'Antiquité tardive", dans ID., Etudes sur les Vies de philosophes de l'Antiquité tardive: Diogène Laërce, Porphyre de Tyr, Eunape de Sardes. Paris: Vrin ["Textes et Traditions"], 2001; p. 3-63; p. 32. Ce pythagoricien possède toutes les caractéristiques de l'homme divin. La philosophie est conçue dans le cadre d'une révélation divine, successive à une purification et à une initiation. Le converti devient membre d'un cercle fermé dont l'objectif est de préserver la pureté des dogmes et en aucune façon de les diffuser. Le maître - qu'il s'agisse d'Apollonius de Tyane ou de Pythagore - occupe une place intermédiaire entre les dieux et les hommes, il forme une troisième espèce, comme l'indique Jamblique, De vita pythagorica, 28.144: "Parmi les bipèdes, il faut compter les hommes, les oiseaux et une troisième espèce [ie Pythagore]".

3333 Cf. BIELER, L. $\Theta E I O \Sigma$ ANHP. Das Bild des göttlichen Menschen in Spätantike und Frühchristentum, Wien 1935-36, 2 vols, cité par R. GoulET, op.cit., p. 26; les caractéristiques du theios anêr sont les suivantes: annonciation de sa naissance, laquelle a lieu dans des conditions exceptionnelles, qualités morales et intellectuelles hors du commun, conversion à la philosophie pythagoricienne, voyage chez les Sages d'Orient, ascétisme (végétarisme), piété.

${ }^{34} \mathrm{Par}$ là nous ne voulons pas dire que Porphyre et Plotin s'écartent complètement de la rationalité. Pour eux en effet, l'accès au divin se fait par un retour de l'âme à l'intelligible, donc pas une forme de raison intuitive dont le modèle ultime est l'extase, éprouvé dans le silence de la pensée pure. Le modèle est alors Pythagore. Le traitement réservé par Diogène à ce dernier nous laisse à penser que ce n'est pas ce modèle de rationalité - opposé aux superstitions et aux sorciers chez Porphyre par exemple - qu'il préconiserait.

${ }^{35}$ Eunape (349 - après 414) signale par ailleurs que la Vie d'Apollonius de Tyane aurait pû s'intituler "Visite d'un dieu chez les hommes": cf. R. GoulET, op. cit., p. 24.
} 
pas la tradition qui voit en Pythagore un homme divin. Loin de multiplier les témoignages sur ses prodiges comme le font Jamblique et dans une moindre mesure Porphyre, il les explique rationnellement: lorsque Pythagore prétend par exemple s'être rendu dans l'Hadès, il note qu'il s'était fait construire une habitation souterraine, suivant en cela sa source Hermippe ${ }^{36}$. De manière cette fois inhabituelle, les épigrammes traitent de points de doctrine, soulignant l'absurdité du végétarisme, le caractère ridicule de la mort de Pythagore ${ }^{37}$, la contradiction qu'il y a à laisser les autres commettre ce que l'on a défini comme injuste (à savoir, manger de la viande ${ }^{38}$ ). Cela laisse supposer que les superstitions, les conduites mystiques seront rejetées au moyen de raisonnements. En témoigne sa dénonciation systématique de ce qui lui apparaît clairement comme des impostures. Citons la présentation de la mort d'Empédocle: parmi les différentes versions ${ }^{39}$, seules deux des versions les plus "naturelles" sont chantées dans les épigrammes, à savoir l'explication de sa mort par des accidents: la chute dans l'Etna et la chute d'un char. De la même façon, Héraclide, exemple même de l'imposteur démasqué, joue au sage mais n'est qu'une bête: il incarne exactement ce qu'il ne faut pas faire. L'exposé de sa Vie se déroule comme se déroulent toutes les autres Vies, sans commentaire et même avec quelques éléments laudatifs ${ }^{40}$. Les choses se gâtent avec l'exposé de ses nombreuses tromperies, toutes dénoncées comme telles. À l'acceptation crédule de certains phénomènes par la foule, Diogène Laërce oppose les explications de ses sources, Démétrios de Magnésie, Hippobote, Hermippe: Héraclide veut faire croire qu'il est ressuscité sous la forme d'un serpent (apprivoisé); il corrompt la Pythie pour se faire rendre des honneurs; il est à chaque fois découvert et le doxographe

\footnotetext{
${ }^{36} \mathrm{DL}, \mathrm{VIII.} 41$.

${ }^{37}$ Alors même qu'il est poursuivi par ses ennemis, il se fait prendre car il refuse de traverser un champ de fèves.

${ }^{38} \mathrm{DL}, \mathrm{VIII.} 44-45$.

${ }^{39}$ DL, VIII. 67-74: Héraclide et Pausanias sont les tenants d'une divinisation d'Empédocle: après avoir ressuscité une femme morte, il s'évapore dans une lumière céleste. Hermippe transmet également l'anecdote d'une guérison miraculeuse. Hippobote et Timée vont s'opposer à cette divinisation, en faisant apparaître des machinations: pour Hippobote, le philosophe aux sandales d'airain ne s'évapore pas mais se jette dans l'Etna pour faire croire à sa divinité; pour Timée, son exil dans le Péloponnèse explique les incertitudes quant aux circonstances de sa mort. Suivent les versions plus prosaïques de Néanthe (il tombe d'un char, se brise le fémur et meurt à Mégare), de Démétrios de Trézène (il se pend), ainsi que celle de la lettre de Telaugès (il tombe et se noie dans la mer).

${ }^{40}$ DL, V. 89: il est varié et noble quant au style et capable à suffisance d'entraîner l'âme de son lecteur; DL, V. 86: il a écrit des traités très beaux et excellents.
} 
conclut: "Mais tu t'es trompé pour avoir rusé: car oui la bête était un serpent, mais toi, on t'a pris à faire la bête, non le sage $»^{41}$.

Est-ce la tromperie, l'usurpation du nom de sage, ou bien plutôt la superstition et la crédulité des foules qui est dénoncée ? Sans doute les deux à la fois. Nous éclairerons cette question en versant au dossier deux éléments complémentaires quant au rapport de Diogène Laërce au religieux.

Le premier se trouve dans une allusion précédant l'épigramme à Théodore, dit l'Athée. Diogène semble admirer ce dernier:

Nous sommes tombés par hasard sur un ouvrage de lui intitulé Sur les dieux, qui ne prête pas au mépris. C'est à ce livre, dit-on, qu'Épicure emprunta la plupart des choses qu'il a dites ${ }^{42}$.

Le surnom de Théodore l'Athée ne doit pas nous égarer, d'autant qu'il porte également le surnom de "Dieu ${ }^{43}$. Théodore croit en l'existence du dieu $^{44}$ mais "rejette absolument les croyances en des dieux ${ }^{45}$. Son personnage, tel qu'il est décrit par Diogène Laërce, est proche des cyniques, mêlant liberté de parole, impertinence envers les rois, contestation des normes. Ce qu'il conteste est donc moins, comme Épicure, l'existence des dieux que notre conception de leur nature et la forme prise par le culte que nous leur rendons.

Le second élément est l'exemple de Bion, exemple particulièrement intéressant tant dans sa présentation que dans les conséquences que l'on peut en tirer quant à la conception laërtienne de la philosophie comme de la religiosité. Le doxographe remarque que:

Dans la conversation, il [Bion] tenait souvent des propos assez irrespectueux envers les dieux, suivant en cela l'exemple de Théodore ${ }^{46}$.

Pourtant, son péché n'est pas là, mais plutôt dans le fait d'avoir renié ses principes, au moment où il allait mourir. C'est ce comportement qui est fustigé dans l'épigramme laërtienne:

Nous aussi nous l'avons blâmé pour la manière dont il cessa de vivre:

Nous avons entendu dire que Bion [...] disait que les dieux ne sont rien en vérité. S'il était resté attaché à son opinion, il

\footnotetext{
${ }^{41}$ DL, V. 90.

${ }^{42} \mathrm{DL}, \mathrm{II} .97$.

${ }^{43} \mathrm{DL}$, II. 86.

${ }^{44} \mathrm{DL}, \mathrm{II} .100$ et 102.

${ }^{45} \mathrm{DL}$, II. 97.

${ }^{46} \mathrm{DL}, \mathrm{IV} .54$.
} 
aurait été normal de dire: "Il a pensé comme il voulait; mal, mais c'était son avis" ${ }^{\prime 7}$.

Ainsi la principale accusation formulée est-elle le reniement des principes et non pas la négation des dieux ou les injures qui leur ont été faites. L'épigramme laisse apercevoir un principe de tolérance, de libéralité et de respect pour les opinions de chacun. Pourtant, ce n'est pas l'opinion de Diogène, qui la qualifie de mauvaise; dans la suite du texte, l'attitude de religiosité extérieure adoptée par Bion continue d'être désapprouvée, définie comme un retour (vain) à la raison - vain, parce qu'il est clair que l'on n'achète pas les dieux comme cela. Diogène Laërce soutient donc une position conservatrice, de respect des dieux et des rites, mais suggère que toute position doctrinale quelle qu'elle soit, pour peu qu'elle soit cohérente avec elle-même, est respectable. Mais il y a plus. Non content d'avoir renié au dernier moment ses principes, Bion aggrave encore son cas: non seulement il a sacrifié aux dieux, lui qui ne les payait que de sarcasmes, non seulement il leur a demandé pardon, mais:

Sans réticence il tendit son cou à une vieille femme pour un charme et il se laissa convaincre d'attacher autour de ses bras des brassards de cuir, et il posa au-dessus de sa porte un nerprun et une branche de laurier ${ }^{48}$, pauvres amulettes qui n'ont rien à voir avec la conviction religieuse et qui se rapprochent plutôt des pratiques superstitieuses et remèdes de bonne fame.

La position de Diogène est claire: toute philosophie se doit d'être conséquente avec elle-même, jusqu'à la mort d'une part, dans l'accord entre paroles et actes, d'autre part. Toute religiosité ne peut être qu'intelligible, éclairée par la raison. Si Diogène ne se prononce jamais explicitement sur la bonne méthode que doit suivre l'esprit dans la définition de ses principes d'existence, il est cependant possible d'en établir quelques éléments, la voie à suivre étant définie en creux par l'exposition de tous les chemins qu'il ne faut pas emprunter. Là où Sextus, Clément et Porphyre désignaient une voie unique de salut, Diogène se contente de nous indiquer une méthode, sans se

${ }^{47} \mathrm{DL}, \mathrm{IV} .55$. C'est nous qui soulignons.

${ }^{48} \mathrm{DL}$, IV. 56-57. Le nerprun est une sorte de prunier noir. 
prononcer pour une doctrine en particulier. Là où Philostrate, Jamblique et Eunape nous conseillaient la voie obscure des mystères pythagoriciens, Diogène préconise la voie rationnelle. Reste à définir ce qu'est exactement cette rationalité.

Avant de poursuivre sur ce point, nous ferons une remarque sous forme d'hypothèse. Il est possible de comprendre les œuvres des auteurs cités plus haut comme des tentatives pour élever des remparts contre le christianisme naissant, en faisant appel aux sagesses du passé. Eunape par exemple veut promouvoir les valeurs intellectuelles et religieuses opposées au christianisme ${ }^{49}$, tandis que Jamblique dresse comme un programme de la sagesse païenne. Il est possible de lire Philostrate et Porphyre sous ce même angle: face à l'avancée chrétienne, les païens auraient développé une stratégie de défense articulée sur la promotion de figures pythagoriciennes ${ }^{50}$. Dans cette optique, Diogène adopterait simplement une stratégie différente, cherchant dans ce que nous appellerions "la rationalité antique" une réponse alternative au pythagorisme.

Cette hypothèse rencontre pourtant quelques limites. La proximité des thèmes traités, en particulier les parallèles possibles avec les Évangiles, peuvent en effet s'expliquer par un fond culturel commun, hypothèse qui semble plus probable: la Vie d'Apollonius de Tyane a été commandée à Philostrate par la femme de Septime Sévère, Julia Domna. À cette époque, on ne connait pas encore de véritable conflit avec les chrétiens ${ }^{51}$ et la tendance est plutôt au renforcement des religions mystériques orientales, en raison de l'origine syrienne de la cour impériale. Il est possible que le pythagorisme soit perçu comme le dernier bastion imprenable des païens face à l'avancée chrétienne, mais ce n'est pas encore le cas à l'époque de Philostrate; il semble

\footnotetext{
${ }^{49}$ Ses œuvres sont composées sur la commande d'intellectuels païens, tels Chrysanthe de Sardes pour ses Vies et Oribase de Pergame pour sa Chronique. II en fait un devoir de piété, en vue de contrecarrer le christianisme.

50 R. Goulet souligne qu' "Apollonius [de Tyane] a d'ailleurs servi aux adversaires du christianisme comme Porphyre et Hiéroclès d'antithèse païenne au Jésus des Chrétiens. On s'est demandé si les similitudes entre le récit de Philostrate et les Evangiles ne s'expliquaient pas déjà par le dessein de contrebalancer l'influence du christianisme. Peut-être ces rapprochements sont-ils dus à la mentalité religieuse populaire commune dans laquelle se sont formées ces traditions": R. GOULET, op. cit., p. 25.

$51 \mathrm{Mgr}$ P. DESCOURTIEUX veut tirer argument de "la violence des persécutions de Septime Sévère dans les années 202-203", op. cit., introduction p. 11. S'il est exact que le prosélytisme juif et chrétien est interdit en 202, il ne semble pas qu'il y ait eu un édit de persécution et le pouvoir impérial semble s'être montré au contraire relativement neutre (cf. M. LE GLAY \& ALII. Histoire romaine, Paris, PUF ["Premier Cycle"] 1991, p. 403-404).
} 
clair en revanche que cette progression des formes orientales du religieux pourrait expliquer la réticence de Diogène Laërce à l'égard des explications où le merveilleux prime, et sa tendance à privilégier les causes sinon rationnelles, du moins naturelles. La fin du deuxième siècle et la seconde moitié du troisième, marquées par des crises internes et externes, constituent des phases d'instabilité politique majeure, avant le nouvel ordre mis en place par Dioclétien en 285. L'immensité de l'Empire suscite révoltes et invasions, favorisées par les difficultés de succession. On voit ainsi des militaires d'obscure naissance accéder au titre d'empereur, tandis que l'influence orientale se renforce par l'accession au pouvoir de Septime Sévère. Il est d'usage de faire commencer le III ${ }^{\mathrm{e}}$ s. en 192, avec l'assassinat de Commode, le dernier des Antonins, qui marque le début de "l'empire de fer rouillé", comme le nomme Dion Cassius. Cette période est marquée par la fin du pouvoir des grandes familles, la contestation du pouvoir de Rome par les provinces et la progression des superstitions et des religions à mystères. La modification de l'art pictural en est un signe: on y figure pour la première fois l'angoisse morale, l'expression prime sur la cohésion organique, l'élément irrationnel et émotionnel prime sur la beauté de la forme. Toutes ces modifications représentatives d'une époque sont déterminantes, mais ne peuvent hélas trouver place ici. Nous citerons simplement la conclusion de Bianchi Bandinelli, en renvoyant à son ouvrage: "De toutes parts surgit la lutte contre la raison, et avec la raison disparaît, pour des siècles, la pensée scientifique ${ }^{52}$. C'est l'héritage grec tout entier qui est nié: les religions à mystères promettent l'accession à une vie bienheureuse dans l'au-delà à des fidèles qui ont été initiés au langage secret et mystérieux du Dieu, dans le plus grand secret et par des hommes voués entièrement à cette fonction. Il est nécessaire de se convertir, donc de faire un choix personnel décisif, qui fait entrer dans une communauté en rupture avec la communauté civile et les rites officiels. Le formalisme des rites traditionnels, leur lien constant au politique et au juridique ne remplit plus le besoin de secret, de connivence et de catharsis comblé par les cérémonies exaltées, bruyantes, furieuses des

\footnotetext{
52 BIANCHI BANDINELLI, R. Rome. La fin de l'art antique: l'art de l'empire romain de Septime Sévère à Théodose 1er, traduit de l'italien par J. C. \& E. Picard. Paris: Gallimard, ["L'Univers des Formes"], 1970, p. 16. Cf. également ID., Rome. Le centre du pouvoir: l'art romain des origines à la fin du Ile siècle, traduit de I'italien par M. C. Guillaume. Paris: Gallimard, ["L'Univers des Formes"], 1969.
} 
religions à mystères ${ }^{53}$. Il reste que l'orientalisation des cultes est plus ou moins claire: elle existe depuis longtemps, mais l'on note un renforcement relatif des cultes d'Isis, Sérapis, Cybèle, divinités d'origine syriennes, ainsi que de la tendance, sous les règnes d'Elagabal et de Sévère Alexandre, à penser une divinité unique ${ }^{54}$ source de toutes les autres, dont le soubassement théorique était déjà fourni par le stoïcisme.

Dans ce contexte, la doxographie devait assumer une charge non pas nouvelle mais renouvelée pourrions-nous dire: chercher dans le passé des modèles d'existence adaptés aux nouvelles conditions politiques et religieuses. Bien des doxographes tendent à chercher un point de rencontre entre les philosophies passées et l'orientalisation religieuse du présent - et le trouvent dans le Pythagorisme - alors que Diogène semble résister à cette pente: pour lui, ce n'est pas dans cette nouvelle conception de la religion qu'est le salut, mais dans le retour à la Raison antique. La doxographie assume alors un rôle primordial: réaffirmer la pluralité des chemins possibles pour l'esprit; définir ces chemins possibles par leur rationalité, i.e. le fait que l'on puisse les expliquer, en rendre raison, les comprendre sans qu'il soit besoin d'une quelconque initiation ou d'un rapport privilégié à une entité divine définie par son inintelligibilité et son inaccessibilité radicales; revendiquer, par conséquent, la toute puissance de l'esprit humain et une confiance absolue dans la faculté humaine de bien juger. L'exemple de Bion nous rappelle que bien juger n'est pas se décider pour le système le plus vrai, mais être capable de rendre compte de ses convictions et surtout être toujours en accord avec elles. Une vie de cohérence en quelque sorte. Peut-être alors pourrons-nous comprendre pourquoi ce sont les Maximes d'Épicure qui sont citées comme achèvement de l'ouvrage, horizon dernier vers lequel il faut tendre:

Eh bien, plaçons maintenant sa couronne, comme on pourrait dire, à l'ensemble de l'ouvrage et à la vie du philosophe, en reproduisant ses Maximes Capitales, et en

\footnotetext{
53 Parmi les religions orientales qui promettent la vie dans l'au-delà, les chrétiens sont les seuls à être persécutés car ils mettent en question l'éternité et le caractère absolu de l'Empire (une autre éternité et universalité est possible) et refusent le culte de l'empereur.

${ }^{54}$ Élagabal impose Hélios ou Sol comme dieu unique, dont les autres figures divines sont l'expression; sous Sévère Alexandre, ce syncrétisme place tous les dieux sur le même plan, puisqu'ils sont tous compris comme le reflet d'une divinité supérieure que les philosophes du IIIe s chercheront à définir.
} 
refermant avec elles l'ensemble de l'ouvrage - pour faire usage à la fin de ce qui est le principe du bonheur ${ }^{55}$.

La philosophie épicurienne est clairement désignée comme l'aboutissement de l'œuvre, du projet laërtien, lui-même explicite ici: il s'agit de fournir des exemples de mode de vie, des exemples concrets et pratiques de rationalité philosophique, dont les Maximes capitales constituent le condensé génial. Pourquoi alors n’avoir pas simplement rédigé un traité épicurien ? Parce que l'objectif n'est pas là. Après avoir souligné la pluralité des chemins possibles, Diogène n'indique ici que sa préférence pour la forme épicurienne mais laisse le lecteur choisir, en ayant cependant saupoudré son ouvrage d'avertissements plus ou moins explicites. Mais là n'est pas le plus important: la convocation des textes épicuriens peut être lue, selon nous, comme le signe d'un élément primordial de la vision laërtienne. Il est clair que Diogène admire beaucoup Épicure; il veut également le défendre contre les attaques personnelles et doctrinales dont il est l'objet; ayant insisté sur ses qualités humaines, il est conscient de la faiblesse de l'argument et ajoute: "Mais nous verrons mieux cela, si nous avançons en prenant appui sur ses doctrines et ses paroles ${ }^{56}$. Jusqu'ici, il suivait parfaitement sa méthode habituelle, à savoir un exposé doxographique dont on ne sait s'il le fait de mémoire, s'il compile plusieurs sources, ou s'il en recopie une. Il décide brusquement de citer entièrement les Lettres d'Épicure: pourquoi ici et seulement ici emploie-t-il ce procédé ? Pour donner une couronne à son œuvre, certes $^{57}$. Mais également sans doute pour donner accès à un texte que l'on se procure probablement difficilement, rareté qui expliquerait le déferlement de critiques sur une doctrine mal connue, et la multiplication des "fous furieux" dénoncée en DL, X. 9. Son objectif inavoué est alors humaniste avant la lettre: seule la référence au texte lui-même permet de trancher; pour critiquer (et choisir), il faut avant tout avoir les moyens textuels de connaître.

\footnotetext{
${ }^{55} \mathrm{DL}, \mathrm{X} .138$.

${ }^{56} \mathrm{DL}, \mathrm{X} .12$.

${ }^{57}$ Les déflationnistes diront qu'il est simplement tout heureux d'avoir trouvé des textes complets, ce qui est rare. Notre hypothèse est fondée sur notre principe méthodologique énoncé plus haut: même si la rencontre de ces textes est le fait du hasard, Diogène ne peut pas se contenter de les copier sans les comprendre, ou sans en faire plus de cas que cela, c'est-à-dire, qu'il aurait pu recopier à cet endroit n'importe quel texte. Cela est rendu impossible, selon nous, par ce qu'il nous dit explicitement: c'est la couronne de l'ouvrage, ce qui suggère qu'il approuve le contenu des textes qu'il a trouvés, à tel point qu'il leur donne un statut particulier dans son œuvre.
} 


\section{Conclusion}

De tous ces éléments nous pouvons conclure ceci: si Diogène Laërce ne nous indique jamais d'erreur doctrinale, s'il ne livre aucun commentaire critique sur les doctrines exposées, il nous laisse pourtant entendre qu'il existe une méthode mauvaise, une voie qui conduit à l'échec, celle de la superstition, de l'irrationnalité, qu'il désapprouve à tout coup. L'attachement au pouvoir explicatif de la raison implique le rejet systématique du miracle, du surnaturel. Il est peu probable qu'il visait explicitement le christianisme, mais il est en revanche clair que, dans ces conditions, les auteurs chrétiens ne pouvaient trouver place dans son exposition.

Ce n'est pas le seul caractère de son ouvrage, nous l'avons vu. L'autre manière manifeste de se tromper de chemin est de professer quelque chose et d'être incapable de vivre selon ce modèle. Il s'agit alors d'une falsification, d'une erreur profonde en ce que l'on se ment à soi-même. Au sein de la rationalité antique, l'erreur doctrinale n'importe pas, puisqu'elle n'existe pas au sens strict: il n'y a au contraire que des erreurs pratiques. Le requisit de rationalité s'exprime donc également par la nécessité d'une conformité entre les actes et les discours. Dire quelque chose, le professer sans l'appliquer, est une faute logique, une absurdité, dans la mesure où la philosophie n'est pas seulement une doctrine, un dogme, mais surtout un cheminement, l'acte d'un esprit incarné, comme nous le rappelait la définition d'une école choisie par Diogène Laërce. En rédigeant ses Vies, Diogène Laërce veut nous présenter l'accord entre biographie et doxographie, à tel point qu'il est possible de parler chez lui de biodoxographie. La philosophie ne se résume pas à une doctrine, elle est un mode de vie, ce qui n'a rien de très original en soi, mais nous permet de comprendre le premier objectif de l'auteur: ce n'est pas une collection d'opinions qui nous est proposée ici, mais de multiples modèles d'existence, tous également valides, dont la difficulté d'application est soulignée. Les philosophes présentés ne sont ni surhumains ni exceptionnels, mais sujets aux mêmes défauts et faiblesses que nous tous. En ce sens, les Vies ont une nette visée d'édification morale: les doctrines ne sont jamais présentées dans leur caractère abstrait, désincarné, mais toujours comme l'émanation de l'esprit, des choix et du rayonnement d'un individu confronté à la complexité d'un réel qui commence à échapper à toute tentative de représentation rationnelle. 
Cette manière inflationniste de comprendre les Vies nous permet de mieux lire la fin de l'ouvrage, où l'on trouve les Lettres épicuriennes citées in extenso ainsi que les Maximes Capitales, il nous signale par ce biais une conviction primordiale: Diogène Laërce n'est pas simplement un érudit amoureux des textes, il est un homme qui clame haut et fort que c'est par la connaissance, la réflexion, la confrontation rationnelle et la réfutation que l'homme pourra se construire et choisir sa propre voie; l'absence de référence au texte, l'argument d'autorité, le silence, le mystère, ne peut au contraire conduire qu'à l'aveuglement, à la sottise, à la rumeur, et à l'expression des passions et des plus bas instincts de l'homme.

E-mail: christelle. veillard@u-paris10.fr

Recebido: outubro/2009

Aprovado: novembro/2009 